\title{
The Structure of Some Bacteriophages Associated with Male Strains of Escherichia coli
}

\author{
BY D. E. BRADLEY \\ Department of Zoology, University of Edinburgh, West Mains Road, Edinburgh 9
}

(Received 16 January 1964)

\begin{abstract}
SUMMARY
The structure of filamentous phages and RNA-containing phages, both specific for male strains of Escherichia coli, is described in detail. The RNA phages are probably in the form of icosahedra comprising 92 morphological sub-units. The filamentous type consists of a protein coat containing a nucleic acid core which is visible in negative stained preparations. A new morphological type of coliphage isolated on a male strain of $E$. coli is described and the manner in which the tail is attached to the head is shown in electron micrographs. Two temperate phages from male strains are studied in detail; their head sub-units are shown to number 325 and are arranged in the form of an octahedron.
\end{abstract}

\section{INTRODUCTION}

The structures of many different phages specific to Escherichia coli have been

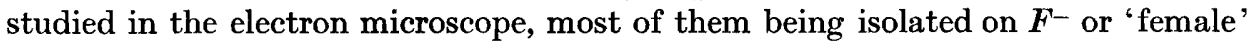
strains of bacteria. The most notable exceptions to this are the ' $f$ ' series which were isolated on $\boldsymbol{F}^{+}$and $\boldsymbol{H} f r$ strains and found to be specific to them. The majority of the series were small 'spherical' types containing ribonucleic acid (RNA; Loeb \& Zinder, 1961) but one of them was a filamentous form containing single stranded deoxyribonucleic acid (DNA; Zinder, Valentine, Roger \& Stoeckenius, 1963). Phages morphologically similar to the ' $f$ ' series have been isolated elsewhere (Marvin \& Hoffmann-Berling, 1963; Hofschneider, 1963; Bradley, 1964) but preliminary electron micrographs have revealed little structural information. In the present paper, the detailed electron microscopy of these morphological types is described. In addition, a new coliphage, originally isolated on an $\mathrm{Hfr}$ strain of $E$. coli, is studied. This virus also grows on $F^{-}$strains. Though it is conventional in form in that it has a head and a tail, it possesses some interesting structural characteristics. The well-known phage $\lambda$, responsible for the lysogeny of $E$. coli strain $\mathrm{k} 12$, has been well studied. However, many other donor strains of $\boldsymbol{E}$. coli are also lysogenic, and the structures of the homologous phages of three such strains are described.

\section{METHODS}

Bacteriophages and hosts. All the phages described are new isolates, and details of their source locality, morphological types and hosts are given in Table 1.

Isolation, growth and purification of virulent phages. The methods used were the same as those already described (Bradley, 1963 $a$ ). The most suitable host bacterium available for isolating phages specific to donor strains of Escherichia coli 
was $E$. coli $\mathrm{C} 3000\left(F^{+}\right)$kindly supplied by Dr C. A. Hutchison (California Institute of Technology).

Isolation, growth and purification of temperate phages. Most $\boldsymbol{F}^{+}$and $\mathrm{H} f \mathrm{r}$ strains of Escherichia coli appear to be lysogenic. The temperate phages responsible were grown as follows. A $10 \mathrm{ml}$. broth culture containing the donor strain and also the phage sensitive E. coli $\mathrm{c} 2$ (Bradley, $1963 \mathrm{~b}$ ) in equal quantities was incubated at $37^{\circ}$ for 3-4 hr with aeration. The culture was then centrifuged at $8000 \mathrm{~g}$ for $10 \mathrm{~min}$. to sediment the bacteria; vegetative phage particles produced in the mixed culture by the lysogenic strain lysed the sensitive $E$. coli $\mathrm{c} 2$ organisms so that a preparation of reasonably high titre could be obtained in one step. Purification by plaque picking from double agar layer plates with $E$. coli $\mathrm{c} 2$ as the host could then be carried out if necessary.

\section{Table 1. Sources of bacteriophages and hosts}

\begin{tabular}{|c|c|c|c|}
\hline Phage no. & Source & $\begin{array}{l}\text { Host strain of } \\
\text { E. coli }\end{array}$ & Source \\
\hline $\mathrm{ZIK} / \mathbf{1}$ & Marquette, U.S.A. & $\mathbf{C} 3000\left(F^{+}\right)$ & Hutchison \\
\hline $\mathrm{ZG} / \mathbf{1}, \mathbf{Z G} / \mathbf{3}_{\mathrm{A}}$ & Basingstoke, England & $\mathbf{C 3 0 0 0}\left(F^{+}\right)$ & Hutchison \\
\hline $\mathbf{Z J} / \mathbf{1}$ & Pangbourne, England & $\mathrm{C} 3000\left(F^{+}\right)$ & Hutchison \\
\hline $\mathbf{Z J} / 2$ & Pangbourne, England & $\mathbf{P}(\boldsymbol{H} f r)$ & Bishop \\
\hline 3000 & Homologous phage of c 3000 & $\mathrm{C2}\left(\boldsymbol{F}^{-}\right)$ & Kay \\
\hline q & Homologous phage of strain $\mathrm{Q}\left(\mathrm{F}^{+}\right)^{*}$ & $\mathrm{c} 2\left(F^{-}\right)$ & Kay \\
\hline & Homologous phage of strain $\mathrm{w}+(\boldsymbol{H} f r)^{*}$ & $\mathrm{C} 2\left(F^{-}\right)$ & Kay \\
\hline WAK/2 & Vienna & K $12\left(F^{+}\right) \dagger$ & \\
\hline & $\begin{array}{l}\text { * Supplied by Dr Bishop. } \\
\uparrow \text { Details of the original source of this str } \\
\text { Note: For addresses of Drs Bishop, Hutc }\end{array}$ & no longe & \\
\hline
\end{tabular}

Efficiency of plating. This was determined for various phage/host systems by plating samples of a phage suspension with the appropriate host by the double agar layer method and counting the resulting plaques. The host used as a basis for comparison was Escherichia coli $\mathbf{c 3 0 0 0 .}$

The spot test for phage activity. A double agar layer plate of host bacterium was prepared and drops of phage suspension placed on it with a platinum loop. After incubation local clearing where the phage was applied indicated that viable virus particles were present.

Isolation of phage-resistant bacteria. In a bacterial population susceptible to a phage there are always some resistant organisms, which can be isolated by several methods. The following method was used here; the phage against which a resistant mutant was required was spotted on the host bacterium by the spot test method and, after incubation, soft agar from the centre of the cleared area was removed with a loop and streaked on an agar plate. The plate was incubated at $37^{\circ}$ for $18-24$ $\mathrm{hr}$. Single colonies of the resulting bacteria were selected, grown on slopes and tested by the spot test for resistance to the phage in question. A satisfactory clone was retained as the required mutant.

Specimen preparation for electron microscopy. The standard negative contrast method was used throughout. The various embedding chemicals included uranyl acetate, potassium phosphotungstate and sodium phosphomolybdate. The electron microscope magnification was calibrated with Dow Polystyrene latex particles (Dow Chemical Co., Midland, Mich., U.S.A.) 


\section{RESULTS}

\section{Small 'spherical' phages}

Morphology. Three isolates of this type were examined (ZIK/1, ZJ/1, ZG/1); all appeared to be morphologically similar. A low magnification electron micrograph of a negative stained preparation (Pl. 1, fig. 1) shows the general appearance. The virions are some $225 \AA$ in diameter and exhibit a regular hexagonal outline. They also pack into a hexagonal array indicating that they are in the form of regular polyhedra. The phosphotungstate appears to have penetrated the protein coat to some extent, producing a characteristic transparent appearance similar to phage 2 (Loeb \& Zinder, 1961). Occasional empty particles can be found (Pl. 1, fig. 2).

Rare departures from the regular hexagonal outline were noted (Pl. 1, fig. 3, arrowed). One of these is larger than normal $(370 \times 225 \AA)$; abnormally large heads have been found in other phages (Anderson, 1960; Bradley, 1963b). Near-focus electron micrographs show little fine structure and it is necessary to underfocus the electron microscope considerably to reveal further detail. In Pl. 1, fig. 4, the particles have a generally rougher appearance; those arrowed ' $A$ ' have a few lines across them suggesting an array of capsomeres while the particles ' $B$ ' have a minute but distinct black spot indicating a pore or aperture in the protein coat about $20 \AA$. in diameter. Micrographs at higher magnification revealed more of these features.

Under favourable conditions of negative staining with phosphotungstate the edges of some particles were obviously separated into capsomeres (Pl. 1, fig. 5, arrowed). Only very rarely could anything resembling an array of discrete capsomeres be found on the particle surface. The best examples are shown in Pl. 1, figs. 6 and 7 .

The pore-like structures could be found on quite a high percentage of particles with careful examination. Sometimes they were very obvious as in Pl. 1, figs. 8 and 9, and very occasionally could be found surrounded by nine or ten capsomeres (Pl. 2, figs. 10 and 11). In no case could a particle be found with more than one pore.

In two cases particles were found in the process of breaking up (Pl. 2, figs. 11 and 12). Fibre-like structural elements appear to be passing through a break in the protein coat; they are probably the contents of the virion. Electron micrographs of empty virions showed no further structural detail (Pl. 2, fig. 13). Capsomeres are visible in the micrograph shown at one point (arrowed) indicating that the structures previously described as capsomeres do, in fact, belong to the protein coat, rather than the nucleic acid core. In some preparations of phage $\mathrm{ZIK} / 1$, curious aggregates were found (Pl. 2, fig. 14). These were caused by the adsorption of RNA phage particles to what appeared to be filamentous phages, and their significance will be discussed below. Negative stains other than phosphotungstate revealed no additional structures.

Other characteristics. The close resemblance of both isolates to the RNA-containing phages of Loeb \& Zinder (1961) suggested that they also contained RNA. Analysis of phage ZIK/1 by alkaline hydrolysis and paper chromatography showed that RNA was present and not DNA. It was also found that the presence of RNase $(50 \mu \mathrm{g} . / \mathrm{ml}$.) in the top layer of double agar layer plates inhibited plaque formation 
by all three isolates, confirming the observation by Zinder et al. (1963). It was concluded that the phages of this morphological type contained RNA.

Resistant mutants against ZIK/1 were easily made by the method described. These strains of Escherichia coli were resistant to the other RNA phages and also the filamentous phages described below. Host range tests with all three RNA phage isolates indicated that they would only grow on male strains of $E$. coli as is the case with the 'f' phages. The most suitable host, $E$. coli $\mathrm{c} 3000$, was determined by comparing the efficiency of plating of a number of different $\boldsymbol{F}^{+}$and $\boldsymbol{H} f r$ strains.

The three phages produced clear plaques of varying size and with a characteristic ragged edge. They were capable of lysing broth cultures of host bacteria.

\section{Filamentous phages}

Morphology. A crude preparation from a confluently lysed plate of phage ZJ/2 is shown in Pl. 2, fig. 15. It can be seen to consist of a mass of tangled filaments. A more dilute suspension was prepared to determine the dimensions of the infective unit (Pl. 3, fig. 16). The unit length was determined at $8300 \AA$. A diameter of such small dimensions is difficult to measure accurately in the electron microscope but in some preparations filaments lying side-by-side were found (Pl. 3, fig. 17); measurements from such preparations gave an average diameter of $68 \AA$.

No obvious structure can be seen in the micrographs described so far. There are no appendages on the ends of the filaments in Pl. 3, fig. 16. Further magnification revealed that the ends were more or less pointed (Pl. 3, fig. 18, arrowed). In one case, however, the end of the filament appeared to be breaking up into very thin fibres (Pl. 3, fig. 19, arrowed). With filamentous or rod-shaped viruses one expects the capsomeres to be arranged in screw symmetry showing as cross-striations on the capsid, but in the micrographs shown so far, all that can be seen is a filament of uniform density. However, in a very few phosphotungstate preparations it was possible to find evidence suggesting a helical structure of the capsid ( $\mathrm{Pl}$. 3, figs. 20 and 21, arrowed) in the form of striations with a spacing of 20-30 $\AA$.

Where the virions were only covered with a little negative stain, a very thin dark line was sometimes visible down the centre (Pl. 4, fig. 22) indicating some penetration by the phosphotungstate. The use of various other negative stains such as uranyl acetate and sodium tungstate and molybdate produced no additional information. With sodium phosphomolybdate, however, the central dark line was more obvious (Pl. 4, fig. 23) being about $20 \AA$. in diameter. In Pl. 4, fig. 24, a sodium phosphomolybdate preparation containing a mixture of phages $\mathrm{ZJ} / \mathbf{1}$ (RNA type) and ZJ/2 (filamentous type), both the core and some faint crossstriations can be seen on the filament which has an RNA phage particle adsorbed to it.

Other characteristics. The filamentous phage $\mathrm{ZJ} / 2$ seems to be remarkably stable in that it is viable after treatment with $0.5 \%$ Dupanol (sodium lauryl sulphate; Dupont de Nemours, Wilmington, U.S.A.) but is inactivated at higher concentrations. It will withstand a temperature of $100^{\circ}$ for some time (10\% survival after $8 \mathrm{~min}$.).

Resistant mutants were prepared and found resistant also to other filamentous and RNA phages. An Hfr strain of Escherichia coli (strain P) was found to be the most suitable host determined by comparing the efficiency of plating of this and other strains, but it gave a low efficiency of plating with the RNA phage ZIK/1. The 


$$
F^{+} \text {and Hfr associated coliphages }
$$

presence of RNase or DNase in the top layer agar of a double agar layer plate did not prevent plaque formation, suggesting that the phage $\mathrm{ZJ} / 2$ contained DNA rather than RNA. Dr D. Kay (private communication) and the author find that this phage does not lyse a broth culture of host bacteria, though the phage titre increases steadily. Somewhat veiled plaques are nevertheless readily formed on double agar layer plates so there is no difficulty in counting phage suspensions. Suspensions with a concentration of $10^{12}$ plaque forming units $/ \mathrm{ml}$. or more are viscous, as would be expected with a particle of this shape and size.

\section{Phage $Z G / 3 A$ (new morphological type)}

Morphology. While this phage is conventional in that it has a head and tail (Pl. 4, fig. 25) it is distinguishable from other coliphages because its head shape resembles that of coliphage $\mathbf{T} 2$, i.e. it is slightly longer than it is wide, yet it has a non-contractile tail. In addition, it possesses a collar (Pl. 4, fig. 25, arrowed), unlike other phages with non-contractile tails. This appears as a single dise when the tail is attached to the head, but when isolated tails are found (Pl. 4, fig. 26) two discs are clearly visible. The empty head in $\mathrm{Pl}$. 4, fig. 27, has a disc remaining within the head membrane. There are some fibres attached to the tail tip of the intact particle in the centre of the field of Pl. 4, fig. 26. Cross-striations are not clear even on empty tails, indicating that they are composed of small tightly packed sub-units ( $\mathrm{Pl}$. 4, fig. 28). The length of the phage tail is $1900 \AA$., and the head is $750 \AA$. long and $600 \AA$. wide. No micrographs obtained so far have provided any clear information regarding the geometrical form of the head.

Other characteristics. This morphological type is not specific to 'male' strains of Escherichia coli and grows readily on some $\boldsymbol{F}^{-}$strains. Plaque formation is not inhibited with RNase. The plaques are rather small (less than $1 \mathrm{~mm}$.) and veiled. The particles have been observed adsorbed tail first to bacteria, as might be expected.

\section{Temperate phages}

Morphology. The temperate phages often found associated with 'male' strains of Escherichia coli appear to have a very similar morphology. Three isolates are described here (see Table 1). Phage 3000 appears to be identical with the SBL types $\beta 4$ and $\gamma 2$ (Bradley \& Kay, 1960; Bradley, 1963a). It is shown in Pl. 4, fig. 29. The head shape is uncertain, while the tail has marked striations with a cluster of indistinct fibres at the tip. The head is $\mathbf{5 5 0} \mathbf{A}$. in diameter and the tail 1400 A. long.

Table 2. Host ranges of temperate phages

\begin{tabular}{|c|c|c|c|c|c|c|c|}
\hline \multirow[b]{2}{*}{ Phage } & \multicolumn{7}{|c|}{ Host } \\
\hline & $\mathbf{P}$ & c 2 & $w+$ & $\mathbf{Q}$ & C 3000 & $\mathrm{c} 2 / \mathrm{w}^{*}$ & $\mathrm{c} 2 / \mathrm{q}^{*}$ \\
\hline 3000 & + & + & + & + & - & + & + \\
\hline$q$ & - & + & - & - & - & - & - \\
\hline $\mathbf{w}$ & - & + & - & - & - & - & - \\
\hline
\end{tabular}


Phages $q$ and $w$ are identical but slightly different from phage 3000. They appear to resemble the well-known phage $\lambda$ which is responsible for the lysogeny of Escherichia coli $\mathrm{K} 12$. The particles of phage q shown in Pl. 5, fig. 30, are clearly octahedra, those marked $\mathbf{A}$ exhibiting the characteristic shape particularly well. At higher magnification cross-striations with a spacing of about $40 \AA$. can be seen on the tails (Pl. 5, fig. 31) which are $1300 \AA$. long and about $70 \AA$. in diameter. The tip of the tail is structurally similar to phage F1 (Bradley, 1963a) as shown in Pl. 5, fig. 32 (see also Pl. 5, fig. 35); this is an easily recognizable and characteristic arrangement of tail fibres. Plate 5, fig. 32, also shows evidence of capsomere structure in the head in the form of rather indistinct lines. The individual units are much clearer in empty heads (Pl. 5, figs. 33 and 34), being about $60 \AA$. in size.

Occasionally unidentified particles resembling empty phage heads can be found in suspensions of this morphological type. The particles are always without tails, sometimes show a hexagonal outline, and are markedly smaller than the phage heads (450 A.). They are shown in Pl. 5, fig. 30, and are marked B.

Other characteristics. The host ranges of these phages are of particular significance. While they are temperate to many male bacteria, they are virulent to other male types and also to most female types. Table 2 shows the results of spot tests on a number of bacteria with the phages examined here. Phages $q$ and $w$ appear similar but 3000 has a wider range.

\section{DISCUSSION}

The RNA phages. It is clear that all the RNA phages isolated so far are morphologically similar. While they superficially resemble the ØX 174 types (Sinsheimer, 1959) they can be easily distinguished on careful examination in the electron microscope. First, the $Ø \mathbf{X} 174$ types are icosahedra with well-marked morphological sub-units on each apex; the RNA types have no such sub-units. The RNA types are penetrated by negative stains whereas the $\emptyset \mathrm{X} 174$ types are not, so that the former appear much more transparent in negative stained preparations than the latter.

A study of the electron micrographs shown provides much basic information on the structure of the virion. The presence of empty particles and particles losing their nucleic acid indicates that the virion is of conventional form in that it consists of a nucleic acid core surrounded by a protein coat. The virions are structurally delicate, their hexagonal outline being easily distorted in negative stained preparations. As a result it is difficult to ascertain the shape and hence the number of capsomeres with absolute certainty. The micrograph (PI. 1, fig. 7) shows the capsomeres in an array and is the clearest obtained so far; the best way of determining the shape of the virion is to compare models of likely figures with it. The icosahedron is the most probable form for many reasons (see Horne \& Wildy, 1961), particularly because it is the commonest figure encountered in virus structure in the cases of very small virions. It also agrees with the predictions of Crick \& Watson (1956). The model to be compared is made up of spherical units to represent the capsomeres since their arrangement will indicate the shape of the whole particle. In Pl. 1, fig. $7 a$ an icosahedron with three sub-units per side is superimposed. It is oriented so as to correspond as nearly as possible to the appearance of the phage particle. A careful 


\section{$F^{+}$and $H$ fr associated coliphages}

study reveals the presence of the 5-fold symmetry axis of the virion in the same position as in the model. Other capsomeres may also be found in the same relative positions. Unfortunately there is a discrepancy in the size of the capsomeres on the model compared to those on the virion. The centre-to-centre spacing on the virion is about $45 \AA$. but is $70 \AA$. on the model. If, however, a model with four sub-units per side is compared ( $\mathrm{Pl}$. 1, fig. $7 b$ ) the centre-to-centre spacing is also about $45 \AA$. Again, a careful comparison between virion and model shows a resemblance perhaps closer than in Pl. 1, fig. 7a. Bearing in mind the fact that the virion has almost certainly been distorted and disorganized, the agreement is good. The inclusion of the two models has been made to illustrate the care required in the interpretation of such electron micrographs. However, one can conclude, with caution, that the RNA phage shown is in the form of an icosahedron, probably with four sub-units per side making a total of $\mathbf{9 2 .}$

One of the most interesting features of the RNA phages is the small pore shown in Pl. 1, figs. 8 and 9, and Pl. 2, figs. 10 and 11. Since there only appears to be one pore per virion, it would seem that it represents a unique site on the capsid. One can only speculate as to its nature; since it appears dark in the micrographs, it is obviously revealed by phosphotungstate penetration. This indicates a discontinuity in the capsid which could only be produced by the absence of a capsomere or by the presence of a hollow or tube-like capsomere. Its diameter of only $20 \AA$. is smaller than the size of the capsomeres so that the second explanation is more likely. Such a structure has not been found on small 'spherical' plant and animal viruses, so that the RNA phages are not morphologically identical to them. They seem to retain a feature common to so many phages: a specific site on the capsid usually associated with adsorption and injection. In most cases this is represented by a tail, but with $\emptyset \mathrm{X} 174$ types it is demonstrated indirectly by the univalence of the phage (Kay, 1962).

A most unusual phenomenon is the adsorption of the RNA phage particles to filamentous phages. The micrograph shown in Pl. 2 , fig. 14, is only a portion of the original which was too big to include as a whole, since it consisted of five filaments attached end-to-end with a total length of about $4 \mu$. In all, $330 \mathrm{RNA}$ phages particles were counted close to or attached to it, giving 66 per unit of filamentous phage. These aggregates were found in mixed cultures of RNA and filamentous phages. They were very infrequent in confluently lysed plates but quite frequent in broth cultures. It is quite possible that the RNA particles are adsorbed to fimbriae rather than filamentous phages though appearance favours the latter, but in either case the result will be to decrease drastically the number of phage particles available for infection. Obviously much more work is required to determine the cause and effect of this phenomenon. It is suggested here that the inhibition of plaque formation by RNA phages using RNase would be a useful test for their quick identification since it operates on all the isolates mentioned here and is probably a general characteristic.

The filamentous phages. The filamentous types so far isolated appear to be morphologically similar though the dimensions given for the different isolates vary somewhat. The length seems to be about 8000 A.; though the individual filaments can stick to one another end-to-end to form very long filaments, these are almost always found to be multiples of $8000 \AA$. in length. The diameters given vary from 
45 to $75 \AA$. Probably most workers have measured the diameters of individual filaments rather than parallel arrays as in the present case. Since the method used here is obviously more accurate, regardless of the negative stain used, the value of $68 \AA$. given is reliable.

The absence of a distinct helical structure on the phage suggests that the capsid consists of small close-packed capsomeres. Even degradation (Pl. 3, fig. 19) reveals them only as indistinct filaments (arrowed) rather than discrete particles. A structure built up in such a manner should be physically and chemically stable, and this is found to be so. According to the figures of Marvin \& Hoffmann-Berling (1963) the virion consists of a single helix of DNA surrounded by protein. Thus the structure is not analogous to that of tobacco mosaic virus where the nucleic acid strand is coiled in a coarse helix within the protein. Here the single DNA helix lies straight up the centre of the filament; in Pl. 4, figs. 22-24, the dark core is visible evidence in support of this. There are no terminal structures for the purpose of adsorption. Indeed, it seems that adsorption does not occur in the truest sense, since the virion passes straight through the cell wall, multiplies within the host, the progeny passing out again without killing the cell. This phenomenon has been demonstrated by electron microscopy (Hofschneider \& Preuss, 1963) and by 'single burst' experiments (Hoffmann-Berling and Mazé, 1964). The release of virions without lysis also occurs with RNA phages below $3^{\circ} \mathrm{C}$. according to the latter authors. Thus the filamentous phages have not only a unique morphology, but also a unique 'life cycle' and many interesting questions are raised. For example, nothing is known about the fate of the capsid after the virion has entered the cell.

Other phages. Two of the remaining phages described have interesting structural features which add to our knowledge of conventional phages in general.

In the first case, phage $\mathrm{ZG} / \mathbf{3}_{\mathrm{A}}$, the manner in which the tail is attached to the head can be clearly seen. There is a single disc or 'washer' on either side of the head membrane securing the tail. This observation immediately suggests an explanation for the small objects found within the empty heads of many phages at the point of attachment of the tail (Bradley \& Kay, 1960), one being illustrated on the arrowed particle in Pl. 5, fig. 31. There are probably similar discs within the head membrane, preventing the tail from becoming detached.

In the cases of phages $q$ and $w$ it is possible to measure the size of the head capsomeres with reasonable accuracy. This combined with the other information given here permits the proposal of a complete model for the entire virion. The head is an octahedron with a side $600 \AA$. long. The capsomeres have a centre-to-centre spacing of $60 \AA$. giving 10 units per side. The total number of capsomeres may be calculated from the formula $4(n-1)^{2}+2$ where $n$ is equal to the number per side. Here, $n=10$ making 326 in all, less one where the tail is attached leaving 325 . The tail is $1300 \AA$. long with $40 \AA$. striations numbering 33 . The tail should have a 4 -fold radial symmetry (Bradley, $1963 b$ ) so that the total number of capsomeres on it will be $4 \times 33$ or 132. Though indistinct, the tail fibres appear to number 5 in Pl. 5, fig. 32, and similar micrographs. The central one is $240 \AA$. long and the side ones $660 \AA$. long with a kink in them. The presence of four side tail fibres fits in with symmetry rule as demonstrated by phage E 1 (Bradley, 1963a). Until this example, it has not been possible to see both the head shape and its capsomeres on a tailed 
bacteriophage. The model given is probably not numerically exact because of the inevitable inaccuracy in measuring small dimensions in the electron microscope, but it is certainly a close approximation.

It is interesting to note that phages $q$ and $w$ have a similar host range (Table 2) and are also morphologically similar. Phage 3000 is slightly different in appearance and has a different host range. It is to be noted from Table 2 that it also attacks mutants of Escherichia coli $\mathrm{c} 2$ which are resistant to phages $\mathrm{q}$ and $\mathrm{w}$ (c2/q and c2/w). Thus it may be stated that $q$ and $w$ are taxonomically similar to one another but are distinct from phage $\mathbf{3 0 0 0}$.

Plate 5, fig. 35, illustrates another morphological type of phage which will grow on male strains of Escherichia coli. It is similar to F1 (Bradley, 1963a) and is clearly an octahedron with a tail structure basically similar to that of phages $q$ and w though the numbers of fibres seems to be different. It is larger than the other two phages.

\section{CONCLUSION}

With the addition of RNA and filamentous viruses to others in the coliphage group it is useful to summarize the present state of knowledge concerning the structure of the various morphological types. This is best achieved by a diagram (Figs. 1-11) and it can be seen that there are now no less than eleven basic morphological types of bacteriophages specific to Escherichia coli. There are still some uncertainties regarding their morphology; the shape of the head of coliphage $\mathbf{T} \mathbf{5}$ is not known for certain but is suspected to be an icosahedron (Bradley, 1963 $a$ ), while the head shapes of the ZG/3A and SBL types are definitely not known. Otherwise there are four examples of octahedra, two icosahedra and one bipyramida hexagonal prism. There is some doubt concerning the number and arrangement of the tail fibres in phages $\mathbf{T} 5$ and $\mathrm{ZG} / 3 \mathrm{~A}$, but the remainder are fairly clear. Most of the phages contain double stranded DNA, two have single-stranded DNA and one RNA.

Though a great deal is known about the structure of these viruses, relatively little is known about their 'life cycle'. Those with contractile sheaths have been well studied and much is known about the method of nucleic acid injection and intracellular multiplication of the $\mathbf{T}$ even types. The remaining coliphages with tails have not been studied extensively and virtually all that is known about their infective process is that they adsorb tail first; the role of their tail fibres is obscure. In the cases of the RNA and ØX 174, types it is not known whether or not they adsorb on to the cell surface in the conventional manner; there is a possibility that the whole virion passes into the cytoplasm, as is the case with filamentous phages. It is obviously important to elucidate fully the mode of infection of the $\emptyset \mathbf{X} 174$ and RNA phages since, like the filamentous types, they closely resemble many plant and animal viruses.

It is of particular interest that the RNA and filamentous phages are specific only to male strains of bacteria. This indicates that the ability to adsorb is connected with the presence of the $F$ agent. Conversely, no ØX 174 type has been found to grow on males, suggesting that the presence of the $\mathbf{F}$ agent prevents adsorption by this morphological type. The fact that male specific phages are common in nature shows that male strains of Escherichia coli are equally common unless they are not 
the natural hosts of the phages, an explanation suggested by Zinder et al. (1963). It is clear from the foregoing that there is still much to be learnt about the coliphages.
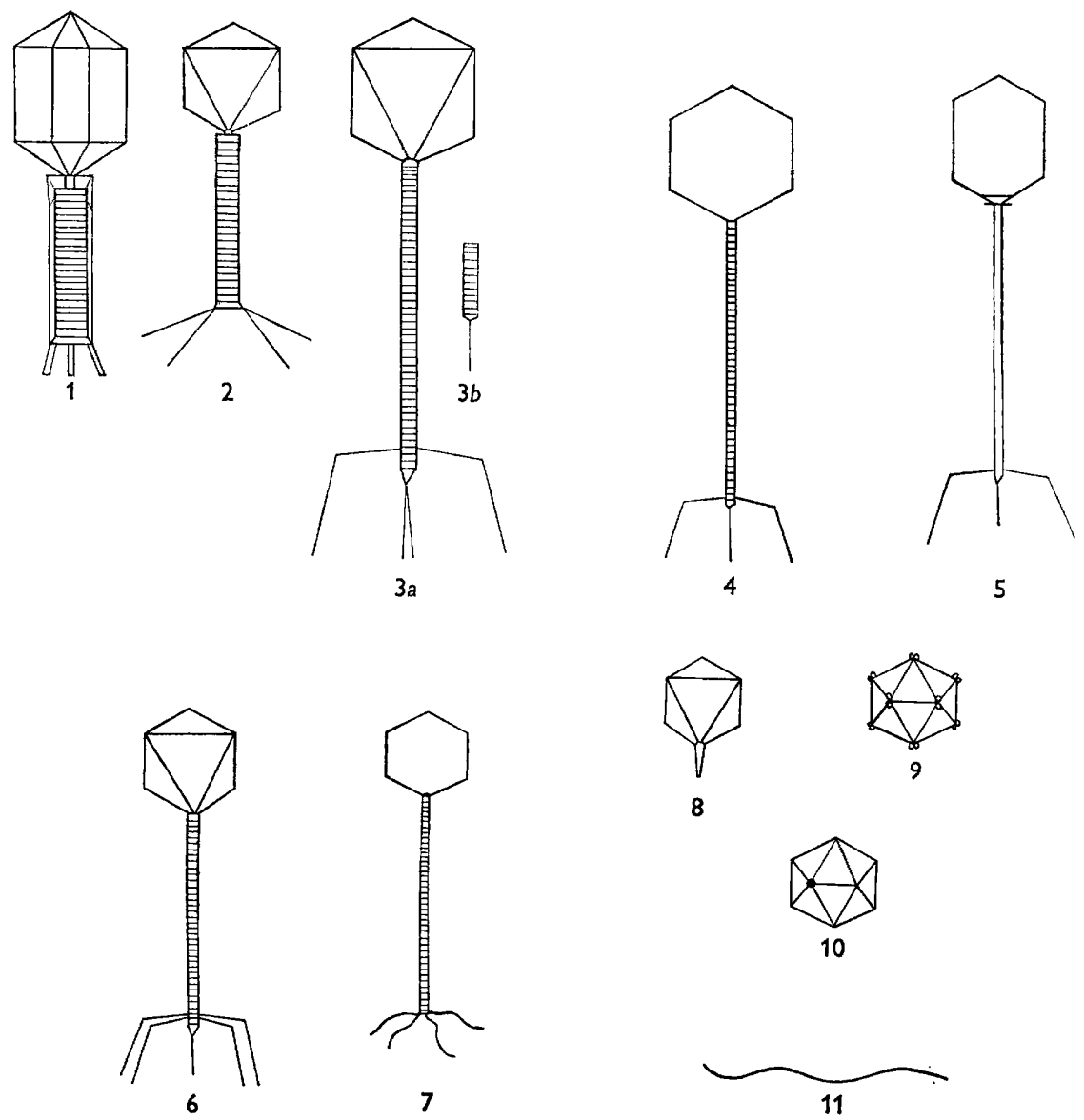

Figs. 1-11. Diagrams of basic morphological types of coliphages.

Fig. 1. T even types, $\times 175,000$. Fig. 2. $\emptyset 2$ types, $\times 175,000$. Fig. 3. $(a)$ F 1 types, (b) tail tip of $\mathrm{Cl}$ types, $\times 175,000$. Fig. 4. T5 types, $\times 175,000$. Fig. 5. ZG/3 A types, $\times 175,000$. Fig. 6. $\lambda$ types, $\times 175,000$. Fig. 7. SBL types, $\times 175,000$. Fig. 8. T3 types, $\times 175,000$. Fig. 9. $Ø X$ types, $\times 385,000$. Fig. 10. RNA phages, $\times 385,000$. Fig. 11 . Filamentous phages, $\times \mathbf{3 8 , 5 0 0}$.

The author would like to thank Dr J. E. Cummins, currently visiting this laboratory, for nucleic acid analysis, Dr D. H. L. Bishop of this laboratory for supplying many male strains of Escherichia coli and for valuable discussion, Dr D. Kay of the University of Oxford, for valuable discussion, Mrs M. Moss and Miss M. McCulloch for technical assistance, and Professor M. M. Swann, F.R.S., for his interest and advice. 


\section{REFERENCES}

Anderson, T. F. (1960). On the fine structure of the temperate bacteriophages P1, P 2 and P 22. Proc. Eur. Reg. Conf. electron microscopy, Delft, 2, 1008.

Bradley, D. E. (1963a). The structure of coliphages. J. gen. Microbiol. 31, 435.

Bradley, D. E. $(\mathbf{1 9 6 3} b)$. The structure of some Staphylococcus and Pseudomonas phages. J. Ultrastructure Res. 8, 552.

Bradley, D. E. (1964). Some preliminary observations on filamentous and RNA bacteriophages. J. Ultrastructure Res. (in the Press).

Bradley, D. E. \& KAy, D. (1960). The fine structure of bacteriophages. J. gen. Microbiol. 23, 553 .

Crick, F. H. C. \& WAtson, J. C. (1956). Structure of small viruses. Nature, Lond. 177, 473.

Hoffmann-Berling, H. \& Mazé, R. (1964). Release of male-specific bacteriophages from surviving host bacteria. Virology, 22, 297.

Hofschneider, P. H. (1963). Untersuchungen über 'kleine' $E$. coli $\mathrm{k} 12$ Bakteriophagen. Z. Naturf. $18 b, 203$.

Hofschneider, P. H. \& Preuss, A. (1963). M 13 bacteriophage liberation from intact bacteria as revealed by the electron microscope. J. molec. Biol. 7, 450 .

Horne, R. W. \& Wildy, P. (1961). Symmetry in virus architecture. Virology, $15,348$.

KAY, D. (1962). The nucleic acid composition of bacteriophage ØR. J. gen. Microbiol. $27,201$.

Loeb, T. \& Zinder, N. D. (1961). A bacteriophage containing RNA. Proc. nat. Acad. Sci., Wash. 47, 286.

Marvin, D. A. \& Hoffmann-Berling, H. (1963). Physical and chemical properties of two new small bacteriophages. Nature, Lond. 197, 517.

Sinsheimer, R. L. (1959). Purification and properties of bacteriophage ØX-174. J. molec. Biol. 1, 37.

Zinder, N. D., Valentine, R. C., Roger, M. \& Stoeckenius, W. (1963). F1, a rodshaped male-specific bacteriophage that contains DNA. Virology, 20, 638.

\section{EXPLANATION OF PLATES}

Plate 1

Fig. 1. Phage ZJ/1 (RNA type) in phosphotungstate. $\times 190,000$.

Figs. 2,3. Phage ZIK/1 (RNA type) in phosphotungstate. $\times 333,000$.

Fig. 4. Phage $\mathrm{ZJ} / \mathbf{1}$ in phosphotungstate. $\times 333,000$.

Fig. 5. Phage $\mathrm{ZJ} / \mathbf{1}$ in phosphotungstate. $\times 500,000$.

Fig. 6. Phage ZG/1 (RNA type) in phosphotungstate. $\times 500,000$.

Fig. 7. Phage ZIK/1 in phosphotungstate showing capsomeres. $\times 500,000 .(a)$ With icosahedron with 42 sub-units; $(b)$ with icosahedron with 92 sub-units.

Figs. 8, 9. Phage ZJ/1 in phosphotungstate showing 'pores'. $\times \mathbf{5 0 0 , 0 0 0}$.

\section{Plate 2}

Fig. 10. Phage ZG/1 in phosphotungstate showing 'pore' surrounded by sub-units. $\times 500,000$.

Fig. 11. Phage $\mathrm{ZIK} / 1$ in phosphotungstate; damaged virion showing pore surrounded by subunits. $\times 500,000$.

Fig. 12. Phage $Z I K / 1$ in phosphotungstate, virion losing nucleic acid. $\times 500,000$.

Fig. 13. Empty virion of phage ZG/1 in phosphotungstate. $\times 500,000$.

Fig. 14. Phage ZIK/1 adsorbed to flament in phosphotungstate. $\times 215,000$.

Fig. 15. Phage ZJ/2 in phosphotungstate. $\times 150,000$. 


\section{Plate 3}

Fig. 16. Phage $\mathrm{ZJ} / 2$ in phosphotungstate. $\times 135,000$.

Figs. 17-20. Phage ZJ/2 in phosphotungstate. $\times 333,000$.

Fig. 21. Phage $\mathbf{Z J} / 2$ in phosphotungstate showing striations. $\times 500,000$.

\section{Plate 4}

Fig. 22. Phage $\mathrm{ZJ} / 2$ in phosphotungstate showing core. $\times 333,000$.

Fig. 23. Phage $\mathrm{ZJ} / 2$ in sodium phosphomolybdate showing core. $\times 333,000$.

Fig. 24. Phages ZJ/1 (RNA type) and ZJ/2 in sodium phosphomolybdate, showing core and striations, $\times 333,000$.

Fig. 25. Phage ZG/3A in phosphotungstate. $\times 333,000$.

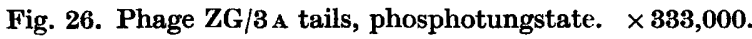

Fig. 27. Phage ZG/3 A empty head, phosphotungstate. $\times 333,000$.

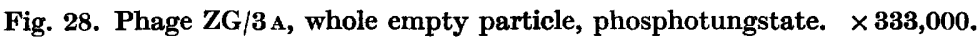

Fig. 29. Phage 3000 in phosphotungstate. $\times \mathbf{3 3 3}, 000$.

\section{Plate 5}

Fig. 30. Phage $q$ in phosphotungstate. $\times 200,000$.

Figs, 31-34. Phage $q$ in phosphotungstate. $\times \mathbf{3 3 3 , 0 0 0}$.

Fig. 35. Phage WAK/2 in phosphotungstate. $\times \mathbf{3 3 3}, 000$.

Note added in proof. The author has recently isolated a ØX174 type phage (St/1, Stoke-on-Trent) which grows on male strains of Escherichia coli, hence this morphological type is not female specific. The author has also observed aggregates of RNA phages (Pl. 2, fig. 14) in pure preparations attached to host cells, thus the virions adsorb to fimbriae rather than filamentous phages. 

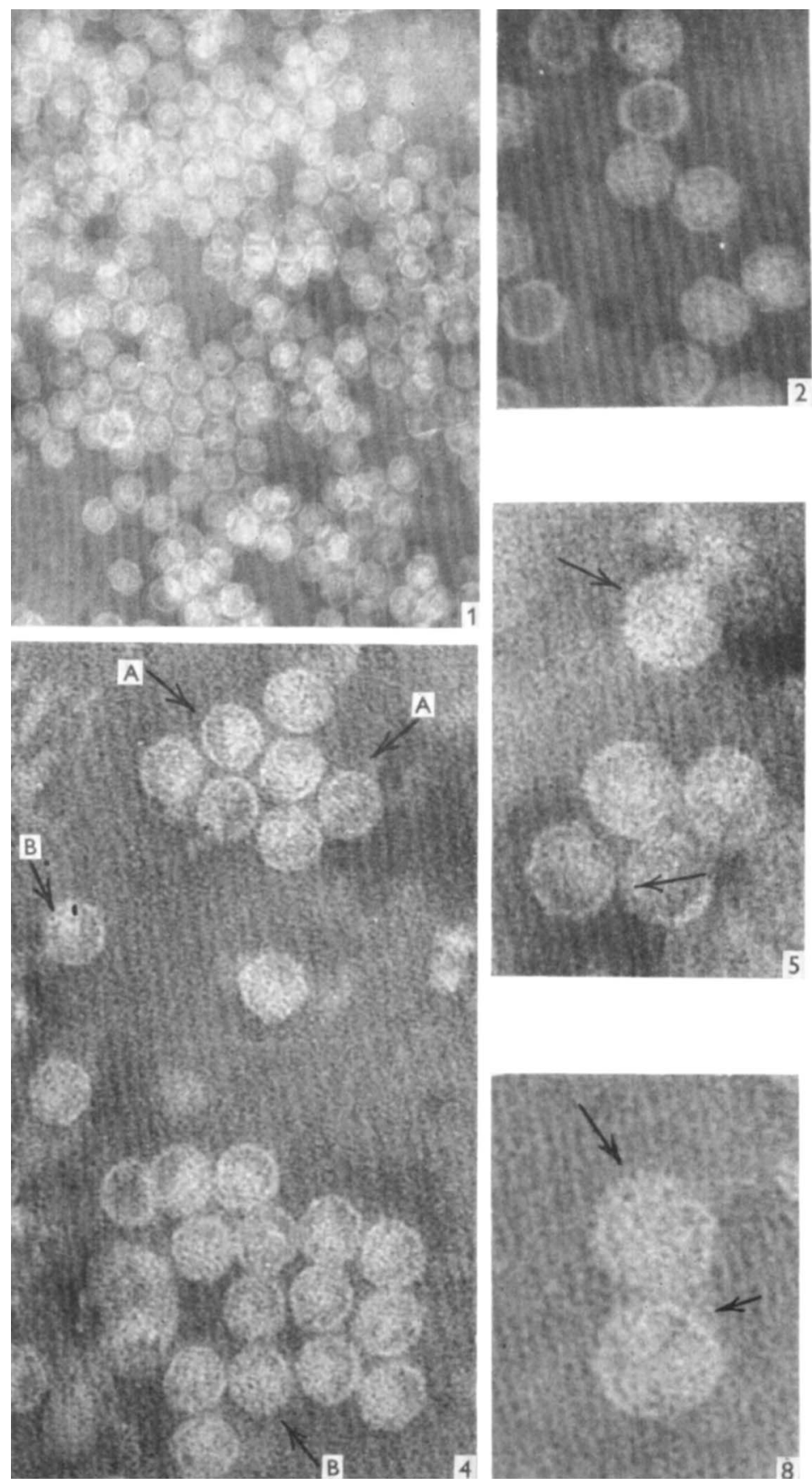
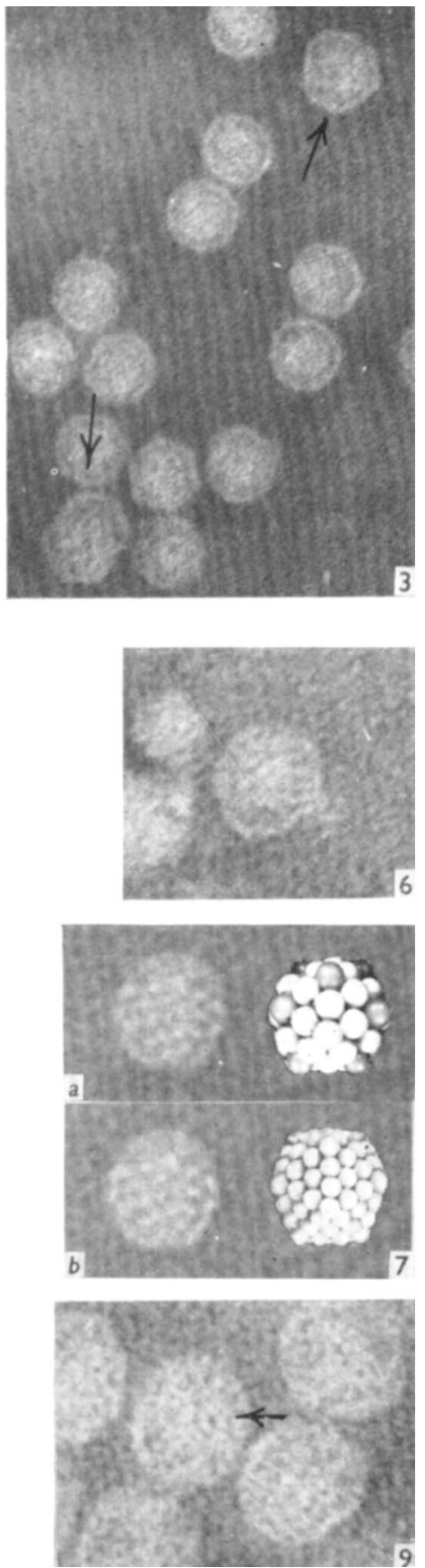

(Facing p. 482)

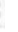

D. E. BRADLEY 
Journal of General Microbiology, Vol. 35, No. 3

Plate 2
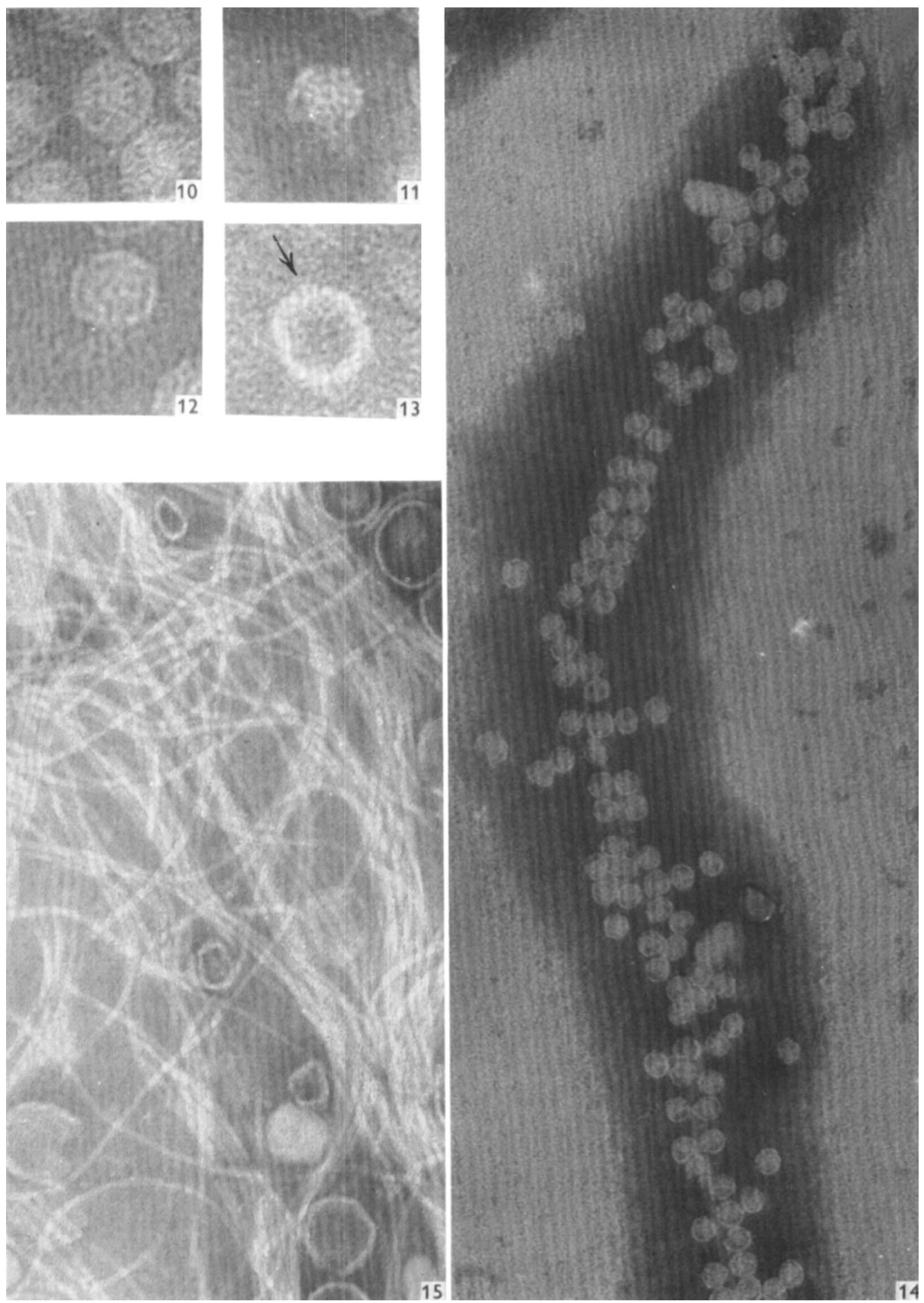

D. E. BRADLEY 

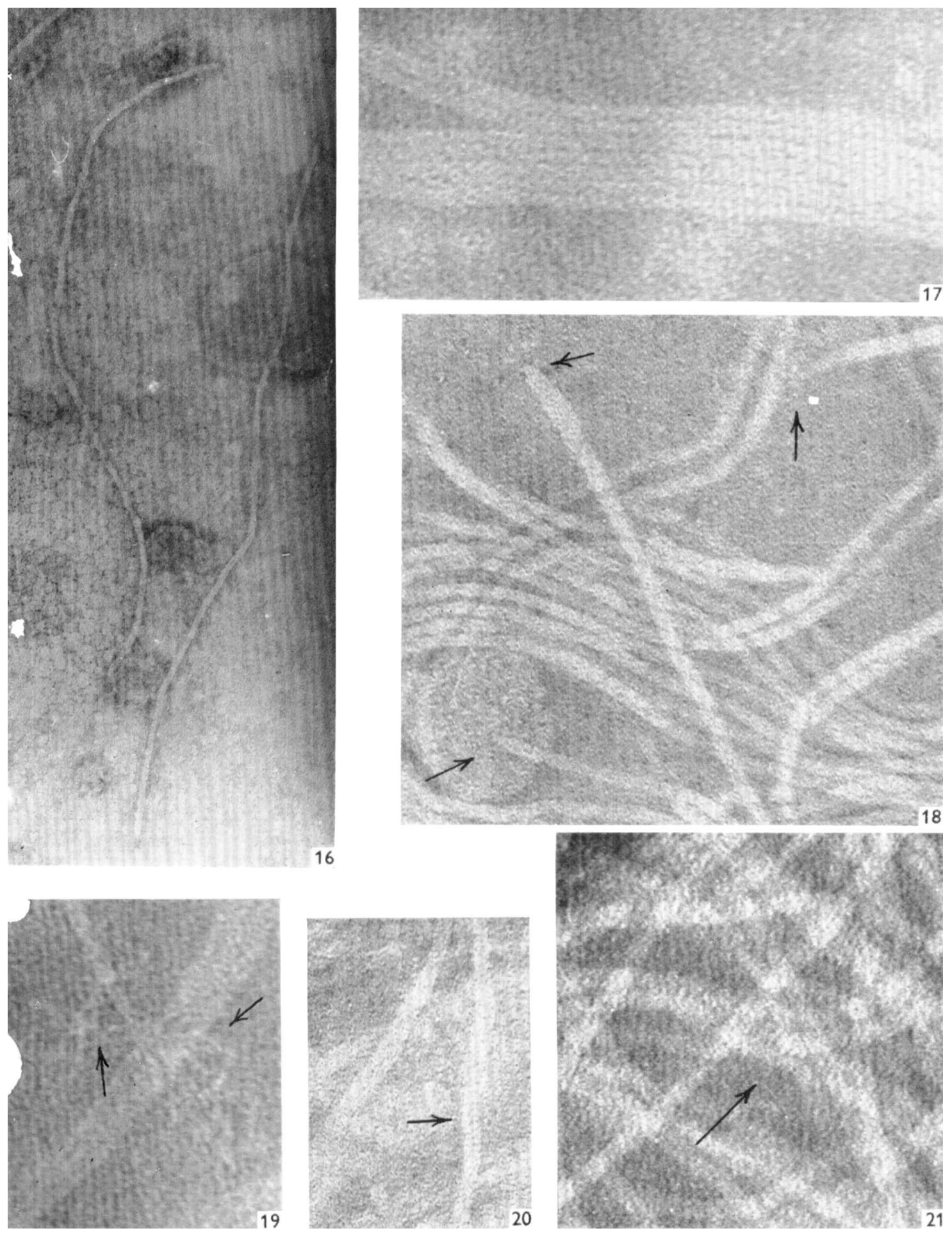

D. E. BRADLEY 
Journal of General Microbiology, Vol. 35, No. 3

Plate 4
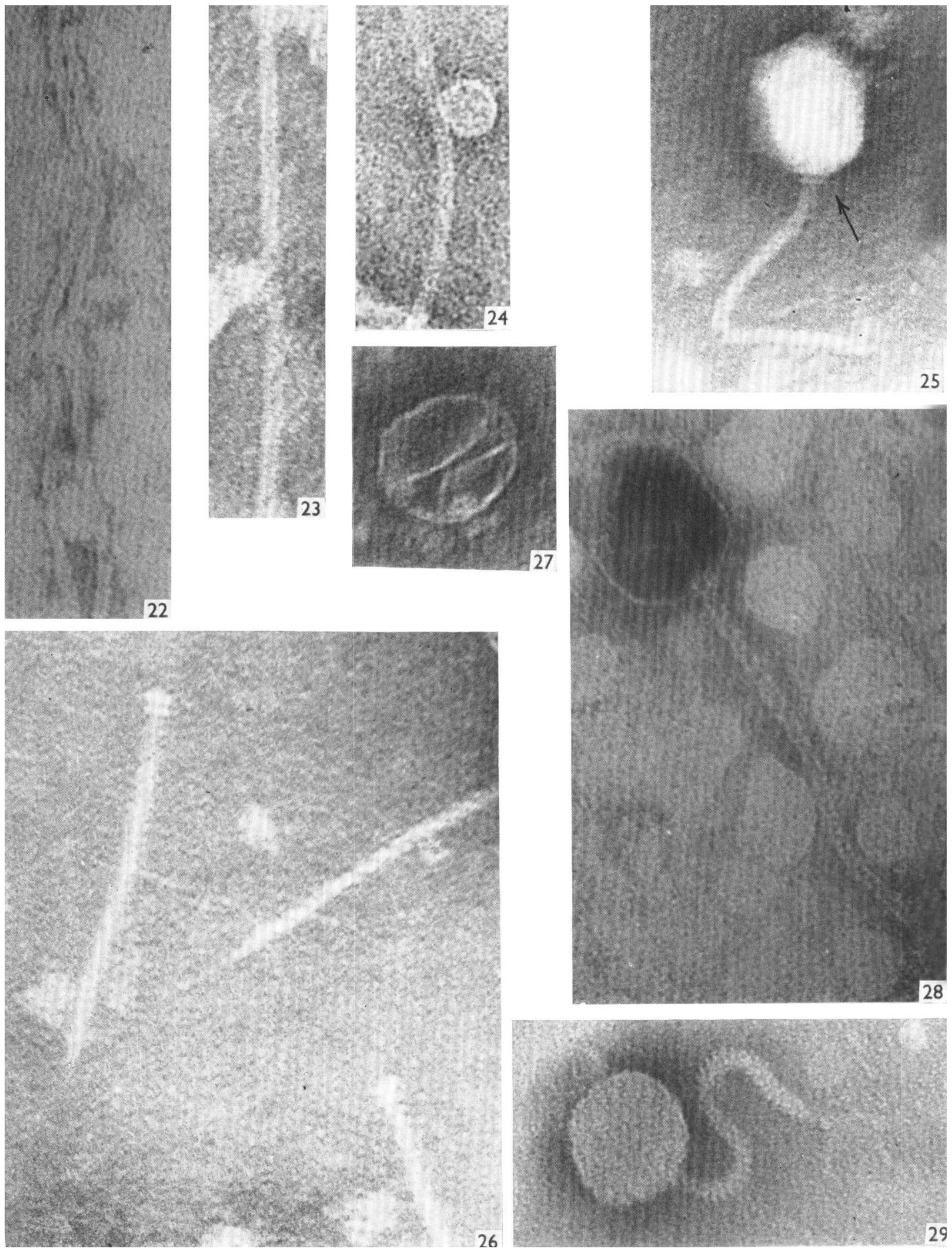

D. E. BRADLEY 

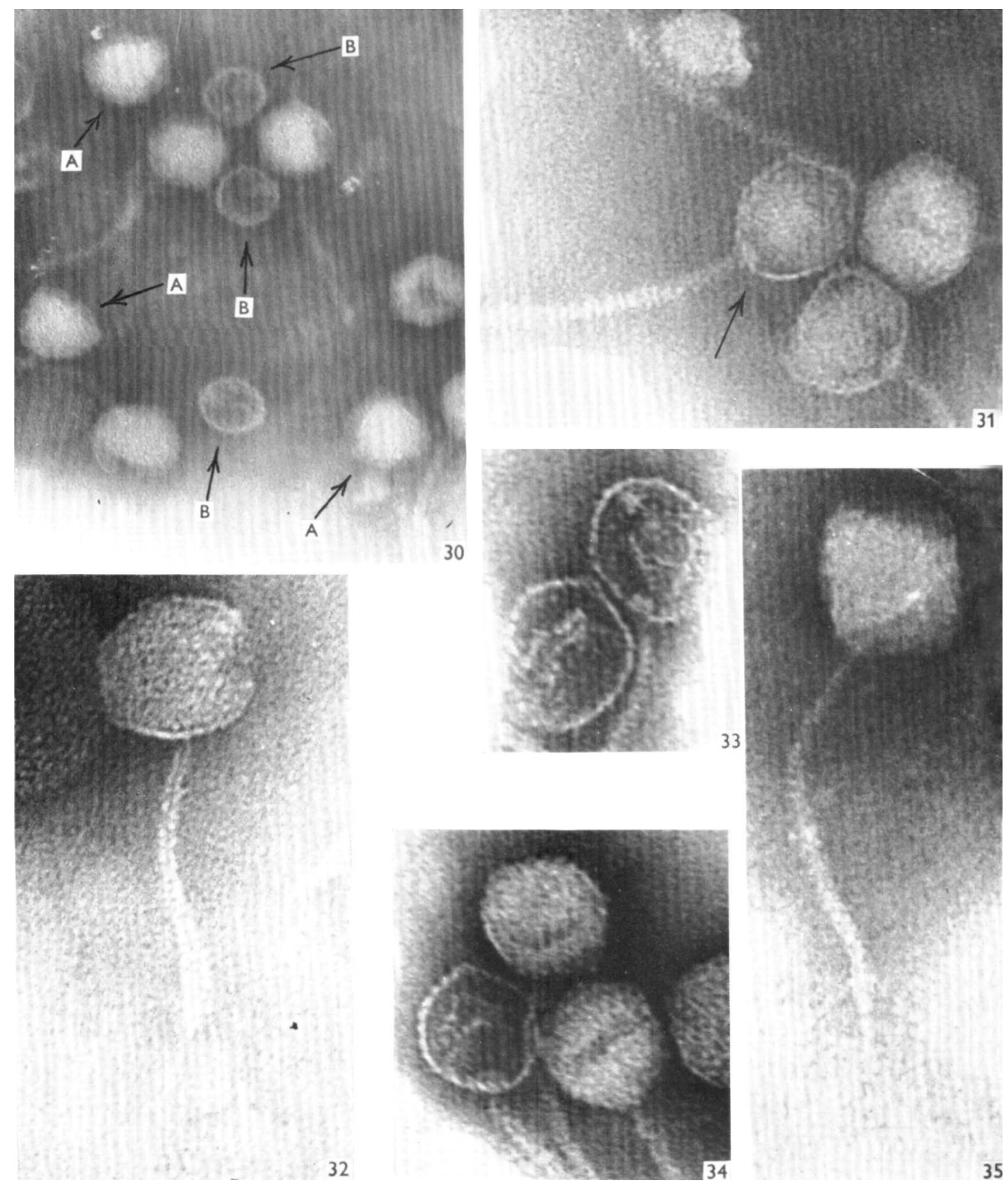

D. E. BRADLEY 